\title{
Robustly Stabilizing Controllers for Dissipative Infinite-Dimensional Systems with Colocated Actuators and Sensors*
}

\author{
Ruth F. Curtain and Job C. Oostveen \\ Department of Mathematics, University of Groningen \\ P.O. Box 800, 9700 AV Groningen, The Netherlands \\ R.F.Curtain@math.rug.nl \\ J.C.Oostveen@math.rug.nl
}

April 14, 1997

\begin{abstract}
We derive formulas for normalized doubly coprime factorizations over $\mathbf{H}_{\infty}$, for a class of nonexponentially stabilizable systems. Using these results, we solve the problem of robust stabilization with respect to normalized coprime factor perturbations for a class of infinite-dimensional systems $\Sigma\left(A, B, B^{*}, D\right)$, with finite-rank inputs and outputs and dissipative $A$; these systems are not exponentially stabilizible We give a parameterization of all controllers which achieve a given robustness margin
\end{abstract}

\section{Introduction}

The problem of robust stabilization with respect to normalized coprime factor perturbations is now a classic $\mathbf{H}_{\infty}$ problem that admits an elegant solution via the solution of a Nehari problem for a normalized left-coprime factorization of the system transfer function. The most general solution was obtained by Georgiou and Smith in [15] for plants possessing a normalized left-coprime factorization. It is known that not all transfer functions possess coprime factorizations, but that stabilizability is a necessary and sufficient condition for this (see Smith [21]). The disadvantage of the latter solution is that, although very general, it does not yield explicit formulas for the robustly stabilizing controllers; These depend on explicit formulas for a normalized doubly-coprime factorization of the transfer function and

\footnotetext{
${ }^{*}$ This research was carried out while the authors were visiting the Center for Research in Scientific Computation, North Carolina State University from January to March, 1997.
} 
these are not available for irrational transfer functions, in general. Special classes for which these are available can be found in Curtain [6], Curtain, Weiss and Weiss [11], Curtain and Zwart [13].

In MacFarlane and Glover [17] the explicit formulas for the normalized coprime factorizations were exploited to obtain explicit formulas for the robustly stabilizing controllers in terms of the control and filter Riccati equations of the original system. This elegant finite-dimensional result was extended to infinite-dimensional systems with bounded inputs and outputs in Curtain and Zwart [12, Chapter 9.4] and to systems with unbounded inputs and outputs in Curtain [6] and Curtain and Pritchard [9]. All of these results assume that the state-space realization is exponentially stabilizable and detectable. In Dym, Georgiou and Smith they solved the problem for a special class of delay systems, using a frequency domain approach (see Dym, Georgiou and Smith [14]).

In this paper we consider a class of state-space systems that are neither exponentially stabilizable nor detectable. Consequently, the existing theory is not applicable. The main ingredients of the proofs in $[6,9,12,17]$ were a Riccati equation theory, explicit formulas for normalized coprime factorizations and an explicit solution of the Nehari problem. Such results are now available for a class of nonexponentially stabilizable systems. Preliminary results on Riccati equations and normalized coprime factorizations were obtained in Curtain and Zwart [13]. More recently, in Curtain and Oostveen [8], a general theory is developed for Riccati equations for nonexponentially stabilizable systems and the same authors solved the Nehari problem for these systems in [7]. Using these results, the robust stabilization problem for our class of systems can be solved. All of the candidate formulas for robustly stabilizing controllers that one would guess by comparison with the exponentially stabilizable and detectable case, derived in Curtain and Zwart [12, Chapter 9.4], make good sense. What we do in this paper is to show that under certain mild (but essential) assumptions these formulas can be proven rigorously.

The class considered here, although mathematically quite special, is often used to model large flexible structures with colocated sensors and actuators (see Balakrishnan [3], Joshi [16], Bailey and Hubbard [1], Chen et al. [5]). It is the classical example of systems that are strongly stabilizable, but not exponentially stabilizable. The main feature is that it may have infinitely many unstable poles, which means that it is not exponentially stabilizable using a finite-rank, bounded controller: Exponentially stabilizable systems with a finite-rank, bounded input have at most finitely many unstable poles (see Curtain and Zwart [12, Theorem 5.2.6]). The state-space description is $\Sigma(A, B, C, D)$, where $A$ is a dissipative operator on the Hilbert space $Z, B \in \mathcal{L}(U, Z)$ and $B=C^{*}, D=D^{*} \geq 0$. The terminology "colocated" for the condition $B=C^{*}$, comes from the fact that the condition arises if the actuators and sensors are implemented at the same location. This class was analyzed in Curtain and van Keulen [10], where they showed that the transfer function was positive real and that the controller $K=-I$ robustly stabilized the system 
with a robustness margin of at least $1 / \sqrt{2}$.

In Section 2 we give a precise mathematical description of the class of systems we consider and we give an example of a flexible beam. In Section 3, formulas for normalized doubly coprime factorizations of transfer functions in this class are derived. In Section 4 we obtain a complete solution of the robust stabilization problem, including

- a formula for the maximal robustness margin $\varepsilon_{\max }$

- explicit formulas for all robustly stabilizing controllers achieving a robustness $\operatorname{margin} \varepsilon>\varepsilon_{\max }$.

We conclude with a glossary of the notation.

\section{Class of Systems}

We consider bounded linear systems $\Sigma\left(A, B, B^{*}, D\right)$ under the following assumptions.

A1. A generates a $C_{0}$ semigroup of contractions $T(t)$ on the separable Hilbert space $Z$;

A2. $U$ is a separable Hilbert space and $B \in \mathcal{L}(U, Z)$;

A3. $D=D^{*} \geq 0$;

A4. $\Sigma\left(A,-, B^{*}\right)$ is approximately observable;

A5. $\Sigma(A, B,-)$ is approximately controllable;

A6. A has compact resolvent;

One of the properties we will exploit is the positive real property.

Definition 2.1 A transfer function $G(s)$ is called positive real if

1. $\overline{G(\bar{s})}=G(s)$;

2. $G(s)$ is holomorphic on $\mathbb{C}_{0}^{+}$;

3. $G^{*}(s)+G(s) \geq 0$ on $\overline{\mathbb{C}_{0}^{+}}$.

Lemma 2.2 Consider $\Sigma\left(A, B, B^{*}, D\right)$ satisfy the assumptions $A 1-A 6$, and define $A_{B}:=A-B B^{*}$, then

P1. $\Sigma\left(A, B, B^{*}, D\right)$ has a positive real transfer function 
P2. $B^{*}\left(s I-A_{B}\right)^{-1} z \in \mathbf{H}_{2}(U)$ for all $z \in Z$;

P3. $B^{*}\left(s I-A_{B}^{*}\right)^{-1} z \in \mathbf{H}_{2}(U)$ for all $z \in Z$;

P4. $B^{*}\left(s I-A_{B}\right)^{-1} B \in \mathbf{H}_{\infty}(\mathcal{L}(U))$;

P5. AB generates a strongly stable semigroup

P6. $A_{B}^{*}$ generates a strongly stable semigroup

Proof. That $G(s)$ is holomorphic on $\mathbb{C}_{0}^{+}$, follows from Corollary 3.6 in Pazy [18]. The inequality $G^{*}+G \geq 0$ follows, using the fact that $A$ is dissipative (that $A$ is dissipative is the content of the Lumer-Philips theorem, see Theorem 4.3 in [18]). P2 and P3 were proven as Lemma 2 in Curtain and Zwart [13], only assuming A1 and A2. For P4, we use a result from Curtain and van Keulen [10], which states that if $K$ is a positive real transfer function, then $(I+K)^{-1} \in \mathbf{H}_{\infty}$. Applying this to $K(s)=B^{*}(s I-A)^{-1} B$, we obtain $I-B^{*}\left(s I-A_{B}\right)^{-1} B \in \mathbf{H}_{\infty}(\mathcal{L}(U))$. P5 is proven in Benchimol [4] under the assumptions A1, A2, A5, A6 (see also Curtain and Zwart [13]). By duality, P6 follows from A1, A2, A4, A6.

The above mathematical structure typically arises in models of flexible structures with colocated actuators and sensors. The following beam example is taken from Slemrod [20] and he attributes it to Bailey and Hubbard [1].

Example 2.3 Consider a flexible cantilever beam of length 1. An Euler-Bernoulli model is used to model the transverse vibrations $w(x, t)$ of the beam. A mass is attached on the tip of the beam and on one side of the beam a piezo-electric film is bonded. When a voltage is applied to the film, it will apply a bending moment to the beam. This voltage is the control input for the system, as output the angular velocity of the tip is taken. The following is a suitable model for this system.

$$
\frac{\partial^{4} w}{\partial x^{4}}(x, t)+\frac{\partial^{2} w}{\partial t^{2}}(x, t)=0 \text { for } 0<x<1
$$

with boundary conditions

$$
\begin{aligned}
& w(0, t)=\frac{\partial w}{\partial x}(0, t)=0 \\
& \frac{\partial^{2} w}{\partial x^{2}}(1, t)=-\frac{\partial^{3} w}{\partial t^{2} \partial x}(1, t)+v(t) \\
& \frac{\partial^{3} w}{\partial x^{3}}(1, t)=\frac{\partial^{2} w}{\partial t^{2}}(1, t),
\end{aligned}
$$

and measurement

$$
y(t)=\frac{\partial^{2} w}{\partial t \partial x}(1, t)
$$


To obtain a first-order representation, a number of spaces are needed. The input space $\mathcal{U}=\mathbb{R}$ will serve as output space, as well. The state space is the Hilbert space

$$
\mathcal{H}=\left\{\hat{w} \in H^{2}(0,1) \times L^{2}(0,1) \times \mathbb{R} \times \mathbb{R} \mid w_{1}=\frac{\partial w_{1}}{\partial x}=0 \text { at } x=0\right\}
$$

with the inner product

$$
\langle\hat{v}, \hat{w}\rangle_{\mathcal{H}}=\int_{0}^{1} \frac{\partial^{2} v_{1}}{\partial x^{2}}(x) \frac{\partial^{2} w_{1}}{\partial x^{2}}(x) d x+\int_{0}^{1} v_{2} w_{2} d x+v_{3} w_{3}+v_{4} w_{4},
$$

where $\hat{w}=\operatorname{col}\left(w_{1}(x), w_{2}(x), w_{3}, w_{4}\right) \in \mathcal{H}$. We will define the operators $A: D(A) \subset$ $\mathcal{H} \rightarrow \mathcal{H}$ and $B \in \mathcal{L}(\mathcal{U}, \mathcal{H})$ by

$$
\begin{gathered}
D(A)=\left\{\hat{w} \in H^{4}(0,1) \times H^{2}(0,1) \times \mathbb{R} \times \mathbb{R} \mid w_{2}(0)=\frac{\partial w_{2}}{\partial x}(0)=0,\right. \\
\left.w_{2}(1)=w_{3}, \frac{\partial w_{2}}{\partial x}(1)=w_{4}, w_{1}(0)=\frac{\partial w_{1}}{\partial x}(0)=0\right\} \\
A=\left[\begin{array}{cccc}
0 & I & 0 & 0 \\
-\frac{\partial^{4}}{\partial x^{4}} & 0 & 0 & 0 \\
\left.\frac{\partial^{3}}{\partial x^{3}}\right|_{x=1} & 0 & 0 & 0 \\
-\left.\frac{\partial^{2}}{\partial x^{2}}\right|_{x=1} & 0 & 0 & 0
\end{array}\right], B=\left[\begin{array}{l}
0 \\
0 \\
0 \\
1
\end{array}\right] .
\end{gathered}
$$

The system (1)-(5) can be represented by the abstract differential equation on $\mathcal{H}$,

$$
\begin{aligned}
& \frac{d z}{d t}(t)=A z(t)+B u(t) \\
& y(t)=B^{*} z(t)
\end{aligned}
$$

Slemrod [20] has shown that $A$ is a closed, densely defined, skew-adjoint operator with compact resolvent (In fact, $A$ is invertible and $A^{-1}$ is compact). Furthermore, $A$ generates a $C_{0}$-semigroup of contractions $T(t)$ on $\mathcal{H}$. Slemrod computed the eigenvalues of $A$ and showed that there exists an orthonormal basis in $\mathcal{H}$ of eigenvectors of $A$. The eigenvalues of $A$ are purely imaginairy and of the form $\lambda= \pm i \mu^{2}$, where $\mu$ satisfies the equation

$$
\begin{aligned}
0= & 1+\cos (\mu) \cosh (\mu)+\mu[\sinh (\mu) \cos (\mu)-\cosh (\mu) \sin (\mu)] \\
& -\mu^{3}[\cos (\mu) \sinh (\mu)+\sin (\mu) \cosh (\mu)]+\mu^{4}[1-\cos (\mu) \cosh (\mu)] .
\end{aligned}
$$

Hence, it can be concluded that $A$ is a Riesz-spectral operator (for details on Rieszspectral operators see Section 2.3 in Curtain and Zwart [12]). Slemrod showed that $\Sigma\left(A,-, B^{*}\right)$ is approximately observable. Because $A$ is a Riesz-spectral operator, We can use Theorem 4.2.3 in Curtain and Zwart to show that $\Sigma\left(A,-, B^{*}\right)$ is approximately observable if and only if $\Sigma\left(A^{*}, B,-\right)$ is approximately controllable. In 
our case, $A^{*}=-A$ and $\Sigma\left(A,-, B^{*}\right)$ is approximately observable, so we have that $\Sigma(-A, B,-)$ is approximately controllable. It is an easy application of Theorem 4.2.3 in Curtain and Zwart, that $\Sigma(A, B,-)$ is then approximately controllable, as well.

Summarizing, the model (1)-(5) can be represented by $\Sigma\left(A, B, B^{*}\right)$ as in (6), (7). Furthermore $\Sigma\left(A, B, B^{*}\right)$ satisfies the conditions $A 1-A 6$, and as a consequence, it satisfies the properties P1-P6.

In the remainder of this section we introduce some stability notions, that are useful when studying nonexponentially stabilizable systems. We consider bounded linear systems $\Sigma(A, B, C)$, i.e. $A$ generates a $C_{0}$-semigroup on $Z, B \in \mathcal{L}(U, Z)$ and $C \in \mathcal{L}(Z, Y)$ where $U, Z$ and $Y$ are separable Hilbert spaces.

Definition 2.4 $A C_{0}$-semigroup $T(t)$ on a Hilbert space $Z$ is called strongly stable if

$$
\lim _{t \rightarrow \infty} T(t) z=0 .
$$

To obtain a nice Riccati equation theory the following notion of stability of a system is appropriate.

Definition 2.5 The bounded linear system $\Sigma(A, B, C)$ is strongly stable if

1. A generates a strongly stable $C_{0}$-semigroup $T(t)$;

2. $C(s I-A)^{-1} z \in \mathbf{H}_{2}(Y)$ for all $z \in Z$;

3. $B^{*}\left(s I-A^{*}\right)^{-1} z \in \mathbf{H}_{2}(U)$ for all $z \in Z$;

4. $C(s I-A)^{-1} B \in \mathbf{H}_{\infty}(\mathcal{L}(U, Y))$.

Definition 2.6 The bounded linear system $\Sigma(A, B,-)$ is strongly stabilizable if there exists $F \in \mathcal{L}(Z, U)$ such that $\Sigma\left(A_{F}, B, F\right)$ is a strongly stable bounded linear system, where $A_{F}=A+B F$.

Definition 2.7 The bounded linear system $\Sigma(A, B, C)$ is statically stabilizable if there exists $K \in \mathcal{L}(Y, U)$ such that $\Sigma\left(A_{K C}, B, C\right)$ is a strongly stable bounded linear system, where $A_{K C}=A+B K C$.

As is indicated in the introduction, we need some results on Riccati equations from Oostveen [8]. Let $N \in \mathcal{L}(Y, U), Q=Q^{*} \in \mathcal{L}(Y), R \in \mathcal{L}(U), R=R^{*} \geq \mu I$ for some $\mu>0$ and consider the following Riccati equation for $z \in \mathcal{D}(A)$,

$$
A^{*} X z+X A z-\left(B^{*} X+N C\right)^{*} R^{-1}\left(B^{*} X+N C\right) z+C^{*} Q C z=0 .
$$

Definition 2.8 The operator $X \in \mathcal{L}(Z)$ is a strongly stabilizing solution to the Riccati equation (8), if 
1. $X$ satisfies (8) for all $z \in D(A)$;

2. $\Sigma\left(A_{F_{X}}, B,\left(\begin{array}{c}F_{X} \\ C\end{array}\right)\right.$ is a strongly stable bounded linear system;

where $A_{F_{X}}=A+B F_{X}$ and $F_{X}=-R^{-1}\left(B^{*} X+N C\right)$.

The Popov function $\Pi: j \mathbb{R} \rightarrow \mathcal{L}(U)$ associated with the Riccati equation (8) is defined by

$$
\Pi(j \omega)=R+N G(j \omega)+G(j \omega)^{*} N^{*}+G(j \omega)^{*} Q G(j \omega)
$$

where $G(j \omega)=C(j \omega I-A)^{-1} B$ and $\omega \in \mathbb{R}$.

The following lemma, which is taken from Curtain and Oostveen [8], gives conditions for the existence and uniqueness of a strongly stabilizing solution to (8).

Lemma 2.9 Consider the Riccati equation (8) with $R \geq \mu I>0$ and assume that $\Sigma(A, B, C)$ is statically stabilizable. (8) possesses a unique self-adjoint, strongly stabilizing solution $X \in \mathcal{L}(Z)$ if and only if $\Pi$ is coercive.

\section{Coprime Factorizations}

In this section, we derive formulas for normalized doubly coprime factorizations over $\mathbf{H}_{\infty}$ for our class of positive real systems.

The class of transfer functions we consider have components in the quotient field $\mathbf{H}_{\infty}\left[\mathbf{H}_{\infty}\right]^{-1}$ (see Curtain, Weiss and Weiss [11].

Definition 3.1 Let $G: \mathbb{C}_{0}^{+} \rightarrow \mathcal{L}(U, Y)$. Suppose that there exist $M(s) \in \mathbf{H}_{\infty}(\mathcal{L}(U))$, $N(s) \in \mathbf{H}_{\infty}(\mathcal{L}(U, Y)), \tilde{X}(s) \in \mathbf{H}_{\infty}(\mathcal{L}(U)), \tilde{Y}(s) \in \mathbf{H}_{\infty}(\mathcal{L}(Y, U))$ with $M$ invertible on some right-half plane, such that

$$
\begin{aligned}
& G(s)=N(s) M(s)^{-1} \quad \text { for } s \in \mathbb{C}_{0}^{+}, \\
& \tilde{X}(s) M(s)-\tilde{Y}(s) N(s)=I \text { for } s \in \mathbb{C}_{0}^{+} .
\end{aligned}
$$

We say that $G=N M^{-1}$ is a right-coprime factorization of $G$ over $\mathbf{H}_{\infty}$.

Suppose that there exist $\tilde{M}(s) \in \mathbf{H}_{\infty}(\mathcal{L}(Y)), \tilde{N}(s) \in \mathbf{H}_{\infty}(\mathcal{L}(Y, U)), X(s) \in$ $\mathbf{H}_{\infty}(\mathcal{L}(Y)), Y(s) \in \mathbf{H}_{\infty}(\mathcal{L}(U, Y))$ with $\tilde{M}$ invertible on some right-half plane, such that

$$
\begin{aligned}
& G(s)=\tilde{M}(s)^{-1} \tilde{N}(s) \quad \text { for } s \in \mathbb{C}_{0}^{+}, \\
& \tilde{M}(s) X(s)-\tilde{N}(s) Y(s)=I \text { for } s \in \mathbb{C}_{0}^{+} .
\end{aligned}
$$


We say that $G=\tilde{M}^{-1} \tilde{N}$ is a left-coprime factorization of $G$ over $\mathbf{H}_{\infty}$. If, in addition

$$
\left(\begin{array}{cc}
M & Y \\
N & X
\end{array}\right)^{-1}=\left(\begin{array}{cc}
\tilde{X} & -\tilde{Y} \\
-\tilde{N} & \tilde{M}
\end{array}\right)
$$

holds on $\mathbb{C}_{0}^{+}$, then we say that $G=\tilde{M}^{-1} \tilde{N}=N M^{-1}$ is a doubly coprime factorization over $\mathbf{H}_{\infty}$. If $(M, N)$ satisfies conditions (10), (11) and also

$$
N(j \omega)^{*} N(j \omega)+M(j \omega)^{*} M(j \omega)=I \text { for } \omega \in \mathbb{R},
$$

we say that $G=N M^{-1}$ is a normalized right-coprime factorization. If $(\tilde{M}, \tilde{N})$ satisfies conditions (12), (19) and also

$$
\tilde{N}(j \omega) \tilde{N}(j \omega)^{*}+\tilde{M}(j \omega) \tilde{M}(j \omega)^{*}=I \text { for } \omega \in \mathbb{R},
$$

we say that $G=\tilde{M}^{-1} \tilde{N}$ is a normalized left-coprime factorization. If (10)-(16) all hold, we say that $G=\tilde{M}^{-1} \tilde{N}=N M^{-1}$ is a normalized doubly coprime factorization.

Our first result shows that for all statically stabilizable, bounded linear systems $\Sigma(A, B, C, D)$, we can get normalized factorizations satisfying (14). However, because we are unable to prove that the Bezout factors are in $\mathbf{H}_{\infty}$, the factorizations are in general not coprime.

Theorem 3.2 Assume that $\Sigma(A, B, C)$ and $\Sigma\left(A^{*}, C^{*}, B^{*}\right)$ are statically stabilizable. $G(s)=D+C(s I-A)^{-1} B$ has the factorizations $G=N M^{-1}=\tilde{M}^{-1} \tilde{N}$, satisfying (10)-(16), where

$$
\begin{aligned}
M(s) & =S^{-\frac{1}{2}}+F_{Q}\left(s I-A_{Q}\right)^{-1} B S^{-\frac{1}{2}} \\
N(s) & =D S^{-\frac{1}{2}}+\left(C+D F_{Q}\right)\left(s I-A_{Q}\right)^{-1} B S^{-\frac{1}{2}}, \\
X(s) & =R^{\frac{1}{2}}-R^{\frac{1}{2}}\left(C+D F_{Q}\right)\left(s I-A_{Q}\right)^{-1} H_{P} \\
Y(s) & =-R^{\frac{1}{2}} F_{Q}\left(s I-A_{Q}\right)^{-1} H_{P} \\
\tilde{M}(s) & =R^{-\frac{1}{2}}+R^{-\frac{1}{2}} C\left(s I-A_{P}\right)^{-1} H_{P} \\
\tilde{N}(s) & =R^{-\frac{1}{2}} D+R^{-\frac{1}{2}} C\left(s I-A_{P}\right)^{-1}\left(B+H_{P} D\right) \\
\tilde{X}(s) & =S^{\frac{1}{2}}-S^{\frac{1}{2}} F_{Q}\left(s I-A_{P}\right)^{-1}\left(B+H_{P} D\right) \\
\tilde{Y}(s) & =-S^{\frac{1}{2}} F_{Q}\left(s I-A_{P}\right)^{-1} H_{P},
\end{aligned}
$$

where $R=I+D D^{*}, S=I+D^{*} D, F_{Q}=-S^{-1}\left(B^{*} Q+D^{*} C\right), H_{P}=-\left(P C^{*}+\right.$ $\left.B D^{*}\right) R^{-1}, A_{Q}=A+B F_{Q}, A_{P}=A+H_{P} C$ and $P$ and $Q$ are the unique self-adjoint, strongly stabilizing solutions of

$$
\left(A-B S^{-1} D^{*} C\right) P z+P\left(A-B S^{-1} D^{*} C\right)^{*} z+B S^{-1} B^{*} z-P C^{*} R^{-1} C P z=0(25)
$$


for $z \in D\left(A^{*}\right)$, and

$$
\left(A-B S^{-1} D^{*} C\right)^{*} Q z+Q\left(A-B S^{-1} D^{*} C\right) z+C^{*} R^{-1} C z-Q B S^{-1} B^{*} Q z=0(26)
$$

for $z \in D(A)$. Furthermore, $\tilde{M}(s) \in \mathbf{H}_{\infty}(\mathcal{L}(Y)), \tilde{N}(s) \in \mathbf{H}_{\infty}(\mathcal{L}(Y, U)), M(s) \in$ $\mathbf{H}_{\infty}(\mathcal{L}(U)), N(s) \in \mathbf{H}_{\infty}(\mathcal{L}(U, Y))$ and $\left(\tilde{X}(s)-S^{\frac{1}{2}}\right) u \in \mathbf{H}_{2}(U), Y(s) u \in \mathbf{H}_{2}(Y)$ for all $u \in U,\left(X(s)-R^{\frac{1}{2}}\right) y \in \mathbf{H}_{2}(Y), \tilde{Y}(s) y \in \mathbf{H}_{2}(U)$ for all $y \in Y$.

Proof. a. First we show that under our assumptions, the Riccati equations (25) and (26) have strongly stabilizing solutions $P$ and $Q . R=I+D D^{*} \geq I$, $S=I+D^{*} D \geq I$, and the Popov functions for (25) and (26) are given by

$$
\begin{aligned}
\Pi_{P}= & R+D^{*} B^{*}\left(j \omega-A^{*}\right)^{-1} C^{*}+C(-j \omega-A)^{-1} B D+ \\
& C(-j \omega-A)^{-1} B B^{*}\left(j \omega-A^{*}\right)^{-1} C^{*} \\
= & I+\left(D+C(-j \omega-A)^{-1} B\right)\left(D^{*}+B^{*}\left(j \omega-A^{*}\right)^{-1} C^{*}\right) \geq I
\end{aligned}
$$

and

$$
\begin{aligned}
\Pi_{Q}= & S+D^{*} C(j \omega-A)^{-1} B+B^{*}\left(-j \omega-A^{*}\right)^{-1} C^{*} D+ \\
& B^{*}\left(-j \omega-A^{*}\right)^{-1} C^{*} C(j \omega-A)^{-1} B \\
= & I+\left(D^{*}+B^{*}\left(-j \omega-A^{*}\right)^{-1} C^{*}\right)\left(D+C(j \omega-A)^{-1} B\right) \geq I,
\end{aligned}
$$

respectively. So, by Lemma 2.9 , both Riccati equations have a unique self-adjoint, strongly stabilizing solution.

b. That $M, N, \tilde{M}, \tilde{N}, X, Y, \tilde{X}, \tilde{Y}$ satisfy (10)-(16) is just linear algebra and makes use of the two Riccati equations (see for instance Curtain and Zwart [12], Theorem 7.3.11 and Exercise 7.29).

c. That $M(s), N(s), \tilde{M}(s)$ and $\tilde{N}(s)$ are in $\mathbf{H}_{\infty}$ is a direct consequence of the fact that $P$ and $Q$ are strongly stabilizing solutions of (25), (26) (Use part 2. of Definition 2.8 and part 4. of Definition 2.5). The claims for $X, Y, \tilde{X}$ and $\tilde{Y}$ follow in the same way, now using parts 2. and 3. of Definition 2.5.

We have been unable to show that $X, Y, \tilde{X}$ and $\tilde{Y}$ are in $\mathbf{H}_{\infty}$ due to the terms $F_{Q}\left(s I-A_{Q}\right)^{-1} H_{P}$ and $F_{Q}\left(s I-A_{P}\right)^{-1} H_{P}$ in formulas (19), (20), (23), (24). Consequently, we cannot conclude that the factorization is coprime.

For our class of positive real systems $\Sigma\left(A, B, B^{*}, D\right)$ under the assumptions A5A6, we can find different $X, Y, \tilde{X}, \tilde{Y}$, which together with $M(s), N(s), \tilde{M}(s), \tilde{N}(s)$ from the theorem above, constitute a normalized doubly coprime factorization. We need the following result, which is taken from Curtain and van Keulen [10].

Lemma 3.3 If $G(s)$ is positive real, then

$$
\begin{aligned}
M(s) & =\tilde{M}(s)=(I+G(s))^{-1} \\
N(s) & =\tilde{N}(s)=G(s)(I+G(s))^{-1} \\
X(s) & =\tilde{X}(s)=-Y(s)=-\tilde{Y}(s)=I
\end{aligned}
$$

form the doubly coprime factorizations $G=\tilde{M}^{-1} \tilde{N}=N M^{-1}$. 
Theorem 3.4 Consider the system $\Sigma\left(A, B, B^{*}, D\right)$ under the assumptions $A 1-A 6$. $G(s)=D+B^{*}(s I-A)^{-1} B$ has a normalized doubly coprime factorization $G=$ $\tilde{M}^{-1} \tilde{N}=N M^{-1}$ satisfying (10)-(16), given by

$$
\begin{aligned}
M(s) & =S^{-\frac{1}{2}}+F_{Q}\left(s I-A_{Q}\right)^{-1} B S^{-\frac{1}{2}} \\
N(s) & =D S^{-\frac{1}{2}}+\left(B^{*}+D F_{Q}\right)\left(s I-A_{Q}\right)^{-1} B S^{-\frac{1}{2}}, \\
\tilde{M}(s) & =S^{-\frac{1}{2}}+S^{-\frac{1}{2}} B^{*}\left(s I-A_{P}\right)^{-1} H_{P} \\
\tilde{N}(s) & =S^{-\frac{1}{2}} D+S^{-\frac{1}{2}} B^{*}\left(s I-A_{P}\right)^{-1}\left(B+H_{P} D\right) \\
\tilde{X}(s) & =-\tilde{Y}(s)=(N(s)+M(s))^{-1} \\
X(s) & =-Y(s)=(\tilde{N}(s)+\tilde{M}(s))^{-1}
\end{aligned}
$$

where $S=I+D^{2}, F_{Q}=-S^{-1}\left(B^{*} Q+D B^{*}\right), H_{P}=-(P B+B D) S^{-1}, A_{Q}=$ $A+B F_{Q}, A_{P}=A+H_{P} B^{*}$ and $P$ and $Q$ are the unique self-adjoint, strongly stabilizing solutions of

$$
\left(A-B S^{-1} D B^{*}\right) P+P\left(A-B S^{-1} D B^{*}\right)^{*}+B S^{-1} B^{*}-P B S^{-1} B^{*} P=0
$$

and

$$
\left(A-B S^{-1} D B^{*}\right)^{*} Q+Q\left(A-B S^{-1} D B^{*}\right)+B S^{-1} B^{*}-Q B S^{-1} B^{*} Q=0
$$

Proof. That $\tilde{M}, \tilde{N}, M$ and $N$ are in $\mathbf{H}_{\infty}(\mathcal{L}(U))$ and satisfy (10), (12), (15), (16) follows by applying Theorem 3.2 . To be able to do so, we need that $\Sigma\left(A, B, B^{*}\right)$ and $\Sigma\left(A^{*}, B, B^{*}\right)$ are statically stabilizable. P2-P5 show that $\Sigma\left(A, B, B^{*}\right)$ is statically stabilizable and P2-P4, P6 do so for $\Sigma\left(A^{*}, B, B^{*}\right)$ (Choose $K=-I$ in the definition of static stabilizability). That (11), (13) and (14) are satisfied is clear, once you remember $(31),(32)$. So, it remains to prove that $\tilde{X}(s)=-\tilde{Y}(s), X(s)=-Y(s) \epsilon$ $\mathbf{H}_{\infty}(\mathcal{L}(U))$. We will only do this for $\tilde{X}(s)$, as the proof for $X(s)$ is completely analogous.

$$
\begin{aligned}
M(s)+N(s) & =(I+D) S^{-\frac{1}{2}}+\left(B^{*}+(I+D) F_{Q}\right)\left(s I-A_{Q}\right)^{-1} B S^{-\frac{1}{2}} \\
& =(I+D)\left\{I+\left(F_{Q}+(I+D)^{-1} B^{*}\right)\left(s I-A_{Q}\right)^{-1} B\right\} S^{-\frac{1}{2}} .
\end{aligned}
$$

So,

$$
\begin{aligned}
& S^{-\frac{1}{2}} \tilde{X}(s)(I+D)=S^{-\frac{1}{2}}(M(s)+N(s))^{-1}(I+D) \\
& \quad=I-\left(F_{Q}+(I+D)^{-1} B^{*}\right)\left(s I-A_{Q}+B F_{Q}+B(I+D)^{-1} B^{*}\right)^{-1} B \\
& \quad=I-\left(F_{Q}+(I+D)^{-1} B^{*}\right)\left(s I-A+B(I+D)^{-1} B^{*}\right)^{-1} B
\end{aligned}
$$

Because $D \geq 0$ implies that $(I+D)^{-1}>\varepsilon I$ for some $\varepsilon>0$, we can factor $(I+D)^{-1}=$ $L L^{*}$, with $L$ coercive, hence invertible. We can then apply Lemma 2 of Curtain and Zwart [13] to $A_{B L}=A-B L L^{*} B^{*}=A-B(I+D)^{-1} B^{*}$, to show that for all $z \in Z, \int_{0}^{\infty}\left\|L^{*} B^{*} T_{B L}(t) z\right\|^{2} d t<\infty$. By the Paley-Wiener theorem and using the 
invertibility of $L$, we obtain $B^{*}\left(s I-A^{*}+B(I+D)^{-1} B^{*}\right)^{-1} z \in \mathbf{H}_{2}(U)$ for all $z \in Z$. Consequently,

$$
\left(\tilde{X}^{\dagger}(-s)-S^{\frac{1}{2}}\right) u \in \mathbf{H}_{2}(U) .
$$

Furthermore, $\tilde{X}(s)=(N(s)+M(s))^{-1}=M^{-1}(s)(I+G(s))^{-1}$, so $M(s) \tilde{X}(s)=$ $\left(I+G(s)^{-1}\right.$ and $N(s) \tilde{X}(s)=G(s)\left(I+G(s)^{-1}\right.$. Because $G(s)$ is positive real, we can apply Lemma 3.3 to show that $M(s) \tilde{X}(s), N(s) \tilde{X}(s) \in \mathbf{H}_{\infty}(\mathcal{L}(U))$. From (15), we obtain

$$
\tilde{X}^{*}(j \omega) \tilde{X}(j \omega)=\tilde{X}^{*}(j \omega) N^{*}(j \omega) N(j \omega) \tilde{X}(j \omega)+\tilde{X}^{*}(j \omega) M^{*}(j \omega) M(j \omega) \tilde{X}(j \omega),
$$

which is in $\mathbf{L}_{\infty}(-j \infty, j \infty ; \mathcal{L}(U))$ and so $\tilde{X}^{\dagger}(j \omega) \in \mathbf{L}_{\infty}(-j \infty, j \infty ; \mathcal{L}(U))$. Combining with (35), we obtain that $\tilde{X}(s) \in \mathbf{H}_{\infty}(\mathcal{L}(U))$

\section{Parameterization of Robustly Stabilizing Controllers}

The problem we consider in this section is that of robust stabilization under normalized coprime factor perturbations, for the class of systems $\Sigma\left(A, B, B^{*}, D\right)$ under the assumptions A1-A6, with the additional assumption that $U$ is finite-dimensional. This is necessary because the Nehari results in Curtain and Oostveen [7] are only valid for $U$ finite-dimensional. Our transfer matrices have components in the quotient field $\mathbf{H}_{\infty}\left[\mathbf{H}_{\infty}\right]^{-1}$. We will denote the class of matrices of any size, with components in the field $\mathcal{F}$ by $\mathcal{M} \mathcal{F}$.

By stability of a closed-loop system we shall mean the following.

Definition 4.1 If $G, K \in \mathcal{M} \mathbf{H}_{\infty}\left[\mathbf{H}_{\infty}\right]^{-1}$ we say that $(G, K)$ is input-output stable if

1. $\operatorname{det}(I-G(s) K(s)) \not \equiv 0$ in $\overline{\mathbb{C}_{0}^{+}}$;

2. $S:=(I-G K)^{-1}$, KS, $S G$ an $(I+K S G) \in \mathcal{M} \mathbf{H}_{\infty}$.

We also say that $K$ stabilizes $G$ in the input-output sense.

For the interpretation of this definition consider the block diagram below.

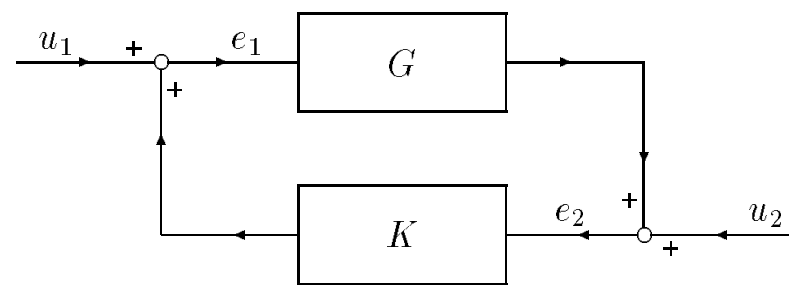


The transfer function from $\left(\begin{array}{l}u_{1} \\ u_{2}\end{array}\right)$ to $\left(\begin{array}{l}e_{1} \\ e_{2}\end{array}\right)$ is

$$
\mathcal{H}(G, K)=\left(\begin{array}{cc}
I+K S G & K S \\
S G & S
\end{array}\right)
$$

Thus, Definition 4.1 states that $(G, K)$ is input-output stable if $K$ stabilizes $G$ in the sense that all paths in the feedback configuration are in $\mathbf{H}_{\infty}$. In Smith [21] it is shown that if $G$ is stabilizable in this sense, then it has a normalized doubly coprime factorization. It is not difficult to show that the converse holds, as well.

For a plant with a transfer matrix in $\mathcal{M} \mathbf{H}_{\infty}\left[\mathbf{H}_{\infty}\right]^{-1}$, possessing a normalized doubly coprime factorization, we consider the following class of perturbations.

Definition 4.2 E-admissible left-coprime factor perturbations of $G$ are plants with transfer matrices given by

$$
G_{\Delta}=\left(\tilde{M}+\Delta_{M}\right)^{-1}\left(\tilde{N}+\Delta_{N}\right),
$$

where $\Delta=\left[\begin{array}{cc}\Delta_{N} & -\Delta_{M}\end{array}\right] \in \mathcal{M} \mathbf{H}_{\infty}$ and $\|\Delta\|_{\infty}<\varepsilon$.

The robust stabilization problem for $G$ we consider, is the problem of finding all $K \in \mathcal{M} \mathbf{H}_{\infty}\left[\mathbf{H}_{\infty}\right]^{-1}$ that stabilize all $\varepsilon$-admissible perturbations of $G$ for a given $\varepsilon>0$. In addition, we are interested in finding the maximal robustness margin $\varepsilon_{\max }$, i.e. the largest $\varepsilon$, for which the above problem has a solution.

Our approach is to adapt the theory of Chapter 9 of Curtain and Zwart [12] to our situation, where we replace exponential stability (stabilizability) by strong stability (stabilizability), and $\mathcal{M} \hat{\mathcal{A}}_{-}$by $\mathcal{M} \mathbf{H}_{\infty}$.

First, we relate the robust stabilization problem to a Nehari problem as in Curtain and Zwart [12], Corollary 9.2.9 and Theorem 9.4.3. For the Nehari problem a parameterization of all solutions has been given in Curtain and Oostveen [7], which will allow us to parameterize all solutions to the robust stabilization problem.

Theorem 4.3 Suppose that $G \in \mathcal{M} \mathbf{H}_{\infty}\left[\mathbf{H}_{\infty}\right]^{-1}$ has a normalized doubly coprime factorization over $\mathbf{H}_{\infty}$. Let $0<\varepsilon<1$ and $K \in \mathcal{M} \mathbf{H}_{\infty}\left[\mathbf{H}_{\infty}\right]^{-1}$. The following four statements are equivalent.

1. $K$ stabilizes all $\varepsilon$-admissible left-coprime factor perturbations of $G$;

2. $K$ stabilizes $G$ and

$$
\left\|\left(\begin{array}{c}
K \\
I
\end{array}\right)(I-G K)^{-1} \tilde{M}^{-1}\right\|_{\infty} \leq \frac{1}{\varepsilon}
$$

3. $K$ has a right-coprime factorization $K=U V^{-1}$ over $\mathbf{H}_{\infty}$ satisfying

$$
\left\|\left(\begin{array}{c}
-\tilde{N}^{\dagger} \\
\tilde{M}^{\dagger}
\end{array}\right)+\left(\begin{array}{c}
U \\
V
\end{array}\right)\right\|_{\infty} \leq\left(1-\varepsilon^{2}\right)^{\frac{1}{2}}
$$


4. There exists $U, V \in \mathcal{M} \mathbf{H}_{\infty}$, such that $\operatorname{det}(V) \neq 0$ on $\mathbb{C}_{0}^{+}$and

$$
\left\|\left[\begin{array}{ll}
-\tilde{N} & \tilde{M}
\end{array}\right]+\left[\begin{array}{cc}
U^{\dagger} & V^{\dagger}
\end{array}\right]\right\|_{\infty} \leq\left(1-\varepsilon^{2}\right)^{\frac{1}{2}}
$$

Proof. $1 \Rightarrow 2$. Suppose that (37) does not hold. We construct a $\Delta \in \mathcal{M} \mathbf{H}_{\infty}$ with $\|\Delta\|_{\infty}<\varepsilon$ such that $G_{\Delta}$ is not stabilized by $K$. Define

$$
F=\left(\begin{array}{c}
K \\
I
\end{array}\right)(I-G K)^{-1} \tilde{M}^{-1}
$$

$\|F\|_{\infty} \geq \varepsilon^{-1}$, so there exists an interval $\left(\omega_{1}, \omega_{2}\right)$ such that $\|F(j \omega)\|>\varepsilon^{-1}$ for all $\omega_{1}<\omega<\omega_{2}$. We choose $\omega_{1}<\omega_{0}<\omega_{2}, z_{0} \in Z,\left\|z_{0}\right\|=1$, such that $\left\|F\left(j \omega_{0}\right) z_{0}\right\|=$ $\delta>\varepsilon^{-1}$ and $\operatorname{det}\left(\tilde{M}\left(j \omega_{0}\right) \neq 0\right.$. Next, define

$$
\Delta(s)=z_{0}\left(F\left(j \omega_{0}\right) z_{0}\right)^{*} \frac{1}{\delta^{2}\left(s+1-j \omega_{0}\right)} .
$$

Then, clearly $\left\|\left.\Delta\right|_{\infty}=\frac{1}{\delta^{2}}\right\| z_{0} z_{0}^{*} F\left(j \omega_{0}\right)^{*}\left\|\leq \frac{1}{\delta^{2}}\right\| z_{0} z_{0}^{*}\|\| F\left(j \omega_{0}\right)^{*} \|=\frac{1}{\delta}<\varepsilon$. Furthermore,

$$
\begin{aligned}
\left(I-\Delta\left(j \omega_{0}\right) F\left(j \omega_{0}\right)\right) z_{0} & =z_{0}-\frac{1}{\delta^{2}} z_{0} z_{0}^{*} F\left(j \omega_{0}\right)^{*} F\left(j \omega_{0}\right) z_{0} \\
& =z_{0}-z_{0}\left(\frac{\mid F\left(j \omega_{0}\right) z_{0} \|^{2}}{\delta^{2}}\right)=0 .
\end{aligned}
$$

Using the identity (see (9.26) in [12])

$$
\operatorname{det}\left(I-G_{\Delta} K\right)=\operatorname{det}(I-G K) \operatorname{det}(\tilde{M})^{-1} \operatorname{det}\left(\tilde{M}+\Delta_{M}\right) \operatorname{det}(I-\Delta F),
$$

we see that $\operatorname{det}\left(I-G_{\Delta} K\right)=0$, and so part 1 of Definition 4.1 is not satisfied; $K$ does not stabilize $G$.

$2 \Rightarrow 1$ is proven completely analogously to the corresponding part of the proof of Theorem 9.2.6 in [12]. (Lemma 9.1.5 is used, but that Lemma also extends without change).

$2 \Leftrightarrow 3 \Leftrightarrow 4$. A straightforward extension of the proof of Theorem 9.4.3 in [12].

This theorem reduces the problem to the solution of the suboptimal Nehari problem for $\tilde{G}=\left[\begin{array}{ll}-\tilde{N} & \tilde{M}\end{array}\right]$, as stated in part 4. The solution to the Nehari problem is well known (see Power [19]):

$$
\inf _{J(-s) \in \mathcal{M} \mathbf{H}_{\infty}}\|\tilde{G}+J\|_{\infty}=\left\|H_{\tilde{G}}\right\|
$$

where $H_{\tilde{G}}$ is the Hankel operator with symbol $\tilde{G}$, defined for $f \in \mathbf{H}_{2}(U \oplus U)$ by

$$
H_{\tilde{G}}: \mathbf{H}_{2}(U \oplus U) \rightarrow \mathbf{H}_{2}(U), \quad H_{\tilde{G}} f=\pi\left(\Lambda_{\tilde{G}} f_{-}\right),
$$


where $\Lambda_{\tilde{G}}$ is the multiplication map induced by $\tilde{G}, f_{-}(s):=f(-s)$ and $\pi$ is the orthogonal projection from $\mathbf{L}_{2}(-j \infty, j \infty ; U)$ onto $\mathbf{H}_{2}(U)$. The difficulty in solving this particular Nehari problem is that, unlike in the case of exponential stability, the Hankel operator is not compact. In Curtain and Oostveen [7], a parameterization of all solutions to the suboptimal Nehari problem at hand (assuming finite-dimensional input and output spaces) was given. We will quote this result, but first we need to introduce to introduce the observability and controllability gramians of $\tilde{G}(s)$. Let $\tilde{G}(s)$ have a realization $\tilde{G}(s)=\tilde{D}+\tilde{C}(s I-\tilde{A})^{-1} \tilde{B}$, and let us for simplicity write $\tilde{U}=U \oplus U, \tilde{Y}=U$ (i.e. $\tilde{G}(s): \tilde{U} \rightarrow \tilde{Y}$ ). The semigroup generated by $\tilde{A}$ on $Z$ is denoted $\tilde{T}(t)$. Let us assume that $\Sigma(\tilde{A}, \tilde{B}, \tilde{C})$ is a strongly stable system

The controllability map $\mathcal{B}: \mathbf{L}_{2}(0, \infty ; \tilde{U}) \rightarrow Z$ is defined by

$$
\mathcal{B} u=\lim _{\tau \rightarrow \infty} \int_{0}^{\tau} \tilde{T}(s) \tilde{B} u(s) d s
$$

and the observability map $\mathcal{C}: Z \rightarrow \mathbf{L}_{2}(0, \infty ; \tilde{Y})$ by

$$
\mathcal{C} z=\tilde{C} \tilde{T}(t) z
$$

The controllability gramian $L_{B}$ and the observability gramian $L_{C}$ are defined by

$$
L_{B}=\mathcal{B B}^{*}, \quad L_{C}=\mathcal{C}^{*} \mathcal{C} .
$$

They are bounded operators on $Z$ and they are the unique self-adjoint solutions to their respective Lyapunov equations.

$$
\begin{aligned}
\tilde{A} L_{B} z+L_{B} \tilde{A}^{*} z & =-\tilde{B} \tilde{B}^{*} z \quad z \in \mathcal{D}\left(\tilde{A}^{*}\right) \\
\tilde{A}^{*} L_{C} z+L_{c} \tilde{A} z & =-\tilde{C}^{*} \tilde{C} z \quad z \in \mathcal{D}(\tilde{A}) .
\end{aligned}
$$

Furthermore, $r\left(L_{B} L_{C}\right)=r\left(L_{C} L_{B}\right)=\left\|H_{\tilde{G}}\right\|^{2}$. See Curtain and Oostveen [7] for the details.

In the case we are interested in, $\tilde{G}=(-\tilde{N}, \tilde{M})=$, where $\tilde{M}, \tilde{N}$ are given by (29), (30). The stability assumption which we made above to assure the boundedness of the controllability and observability gramian are satisfied in this case. This follows ifrom the formulas for $\tilde{N}$ and $\tilde{M}$ and the fact that $P$ is a strongly stabilizing solution of the Riccati equation (33).

We need some technical results for the controllability and observability gramians $L_{B}$ and $L_{C}$.

Lemma 4.4 Let $G$ have a normalized left-coprime factorization $G=\tilde{M}^{-1} \tilde{N}$. The Hankel operator with symbol $\tilde{G}=\left[\begin{array}{ll}-\tilde{N} & \tilde{M}\end{array}\right]$ satisfies $\left\|H_{\tilde{G}}\right\|<1$.

Proof. Clearly, $\left\|H_{\tilde{G}}\right\|=\left\|H_{\tilde{G}_{-}^{\dagger}}\right\| \leq\left\|\Lambda_{\tilde{G}_{-}^{\dagger}}\right\|=1$, where the last equality follows from the fact that for an inner function $F,\left\|\Lambda_{F} f\right\|=\|f\|$. Assume that $\left\|H_{\tilde{G}_{-}^{\dagger}}\right\|=1$. There 
must exist $\left\{f_{n}\right\}_{n \in \mathbb{N}}$ with $f_{n} \in \mathbf{H}_{2}(\tilde{Y}),\left\|f_{n}\right\|=1$ such that $\lim _{n \rightarrow \infty}\left\|H_{\tilde{G}_{-}^{\dagger}} f_{n}\right\|=1$. We define $g_{n}, g_{n}^{+}, g_{n}^{-}$by

$$
\begin{aligned}
g_{n}(j \omega) & =\tilde{G}_{-}^{\dagger}(j \omega) f_{n}(-j \omega) \in \mathbf{L}_{2}(-j \infty, j \infty ; \tilde{U}), \\
g_{n}^{+} & =\pi g_{n} \in \mathbf{H}_{2}(\tilde{U}), \\
g_{n}^{-} & =(I-\pi) g_{n} \in \mathbf{H}_{2}^{-}(\tilde{U}),
\end{aligned}
$$

where $\pi$ is the orthogonal projection from $\mathbf{L}_{2}(-j \infty, j \infty ; \tilde{U})$ onto $\mathbf{H}_{2}(\tilde{U})$. Clearly, $g_{n}^{+}=H_{\tilde{G}_{-}^{\dagger}} f_{n}$ and $\left\|g_{n}^{+}\right\|_{\mathbf{H}_{2}}^{2}+\left\|g_{n}^{-}\right\|_{\mathbf{H}_{2}^{-}}^{2}=\left\|g_{n}\right\|_{\mathbf{L}_{2}}^{2}=1$ (the last equality follows from the fact that for an inner function $F,\left\|\Lambda_{F} f\right\|=\|f\|$ ). Thus, we have $\lim _{n \rightarrow \infty}\left\|g_{n}^{+}\right\|_{\mathbf{H}_{2}}=1$ and $\lim _{n \rightarrow \infty}\left\|g_{n}^{-}\right\|_{\mathbf{H}_{2}^{-}}=0$. For $s=-j \omega$, we have

$$
\left(\begin{array}{c}
\tilde{N}(j \omega)^{*} \\
\tilde{M}(j \omega)^{*}
\end{array}\right) f_{n}(j \omega)=g_{n}^{+}(-j \omega)+g_{n}^{-}(-j \omega) .
$$

Multiplying by $\left[\begin{array}{ll}-Y(j \omega)^{*} & X(j \omega)^{*}\end{array}\right]$, where $X$ and $Y$ satisfy (13), we obtain

$$
f_{n}(j \omega)=\left[\begin{array}{ll}
-Y(j \omega)^{*} & X(j \omega)^{*}
\end{array}\right] g_{n}^{+}(-j \omega)+\left[-Y(j \omega)^{*} X(j \omega)^{*}\right] g_{n}^{-}(-j \omega) .
$$

The left-hand side has an extension to a function in $\mathbf{H}_{2}(\tilde{U})$, the first term on the right-hand side has an extension to a function in $\mathbf{H}_{2}^{-}(\tilde{U})$. and the second term on the right-hand side converges to 0 . This contradiction implies that $\left\|H_{\tilde{G}}\right\| \neq 1$ and therefore $\left\|H_{\tilde{G}}\right\|<1$.

Lemma 4.5 Consider the system $\Sigma\left(A, B, B^{*} D\right)$ with finite-dimensional input space $U$ under the assumptions $A 1-A 6$. Let $\tilde{G}(s)=[-\tilde{N}(s) \quad \tilde{M}(s)]=\tilde{D}+\tilde{C}(s I-$ $\tilde{A})^{-1} \tilde{B}$, where $\tilde{M}$ and $\tilde{N}$ are given by (29), (30). $L_{B}$ and $L_{C}$ are given by

$$
\begin{aligned}
& L_{B}=P \\
& L_{C}=Q(I+P Q)^{-1},
\end{aligned}
$$

where $P$ and $Q$ are as in Theorem 3.4. Then,

$$
r\left(L_{B} L_{C}\right)=r\left(L_{C} L_{B}\right)=r\left(P Q(I+P Q)^{-1}\right) .
$$

Proof. ¿From Lemma 4.4, we obtain that $r\left(L_{B} L_{C}\right)=r\left(L_{C} L_{B}\right)=\left\|H_{\tilde{G}}\right\|<1$. Hence $I-L_{B} L_{C}$ and $I-L_{C} L_{B}$ are boundedly invertible. The proof of Lemma 9.4.10 in [12] applies to prove that $I+P Q$ is boundedly invertible and that (47), (48) hold. The equality of the spectral radii in (49) is then trivial.

We quote the solution of the Nehari problem from Curtain and Oostveen [7].

Theorem 4.6 Let $\tilde{G}(s)=\tilde{C}(s I-\tilde{A})^{-1} \tilde{B}$. The following three statements are equivalent. 
1. There exists a $J(-s) \in \mathcal{M} \mathbf{H}_{\infty}$ such that

$$
\|\tilde{G}+J\|_{\infty}<\sigma
$$

2. $\sigma>r^{\frac{1}{2}}\left(L_{B} L_{C}\right)$

3. There exists an $X(-s) \in \mathcal{M} \mathbf{H}_{2}$ such that $V(-s):=X^{-1}(-s) \in \mathcal{M} \mathbf{H}_{2}$ and $X_{11}^{-1}(-s) \in \mathcal{M} \mathbf{H}_{\infty}$, satisfying the spectral factorization

$$
\left(\begin{array}{cc}
I & \tilde{G} \\
0 & I
\end{array}\right)^{\dagger}\left(\begin{array}{cc}
I & 0 \\
0 & -\sigma^{2} I
\end{array}\right)\left(\begin{array}{cc}
I & \tilde{G} \\
0 & I
\end{array}\right)=X^{\dagger}\left(\begin{array}{cc}
I & 0 \\
0 & -I
\end{array}\right) X
$$

on $s=j \omega, \omega \in \mathbb{R}$.

Moreover, all $J(-s) \in \mathcal{M} \mathbf{H}_{\infty}$ satisfying (50) for $\sigma>r^{\frac{1}{2}}\left(L_{B} L_{C}\right)$ are given by $J(-s)=R_{1}(-s) R_{2}(-s)^{-1}$ with

$$
\left(\begin{array}{c}
R_{1}(-s) \\
R_{2}(-s)
\end{array}\right)=X^{-1}(-s)\left(\begin{array}{c}
Q(-s) \\
I
\end{array}\right)
$$

and $Q(-s) \in \mathcal{M} \mathbf{H}_{\infty}$ satisfies $\|Q\|_{\infty} \leq 1$. An $X$ satisfying (51) and its inverse are given by

$$
\begin{aligned}
& X(s)=\left(\begin{array}{cc}
I & 0 \\
0 & \sigma I
\end{array}\right)+\sigma^{-2}\left(\begin{array}{c}
-\tilde{C} L_{B} \\
\sigma \tilde{B}^{*}
\end{array}\right) N_{\sigma}^{*}\left(s I+\tilde{A}^{*}\right)^{-1}\left(\begin{array}{ll}
\tilde{C}^{*} & \left.L_{C} \tilde{B}\right),
\end{array}\right. \\
& V(s)=\left(\begin{array}{cc}
I & 0 \\
0 & \sigma^{-1} I
\end{array}\right)-\sigma^{-2}\left(\begin{array}{c}
-\tilde{C} L_{B} \\
\tilde{B}^{*}
\end{array}\right)\left(s I+\tilde{A}^{*}\right)^{-1} N_{\sigma}^{*}\left(\begin{array}{ll}
\tilde{C}^{*} & \sigma^{-1} L_{C} \tilde{B}
\end{array}\right),(
\end{aligned}
$$

where $N_{\sigma}=\left(I-\sigma^{-2} L_{B} L_{C}\right)^{-1}$.

Now we can proceed as in Chapter 9 of [12], to obtain a parameterization of all stabilizing controllers for $G(s)$

Theorem 4.7 Consider the robust stabilization problem for $\Sigma\left(A, B, B^{*}, D\right)$ under the assumptions A1-A6, with the additional assumption that $U$ is finite-dimensional.

1. The maximum robustness margin satisfies $\varepsilon_{\max }=\left(1-r\left(P Q(I+P Q)^{-1}\right)\right)^{\frac{1}{2}}$.

2. All robustly stabilizing controllers $K \in \mathcal{M} \mathbf{H}_{\infty}\left[\mathbf{H}_{\infty}\right]^{-1}$ for $G(s)=D+B^{*}(s I-$ $A)^{-1} B$ achieving a robustness margin $0<\varepsilon<\varepsilon_{\max }$. are given by

$$
K(s)=\left[\phi_{11}(s) L(s)+\phi_{12}(s)\right]\left[\phi_{21}(s) L(s)+\phi_{22}(s)\right]^{-1},
$$


where

$$
\begin{aligned}
& \left(\begin{array}{ll}
\phi_{11} & \phi_{12} \\
\phi_{21} & \phi_{22}
\end{array}\right)=\left(\begin{array}{cc}
\frac{1}{\varepsilon} S^{-\frac{1}{2}} & \frac{1}{\sigma} D S^{-\frac{1}{2}} \\
\frac{1}{\varepsilon} D S^{-\frac{1}{2}} & -\frac{1}{\sigma} S^{-\frac{1}{2}}
\end{array}\right) \\
& +\frac{1}{\sigma^{2}}\left(\begin{array}{c}
F_{Q} \\
B^{*}+D F_{Q}
\end{array}\right)\left(s I-A_{Q}\right)^{-1} W^{*}\left(\begin{array}{cc}
\frac{1}{\varepsilon} B S^{-\frac{1}{2}} & -\frac{1}{\sigma} P B S^{-\frac{1}{2}}
\end{array}\right),
\end{aligned}
$$

where $W=\left(I+\left(1-\sigma^{-2}\right) P Q\right)^{-1}, S=I+D^{2}, F_{Q}=-S^{-1}\left(D B^{*}+B^{*} Q\right)$, $\sigma=\left(1-\varepsilon^{2}\right)^{\frac{1}{2}}$ and $P$ and $Q$ are the unique self-adjoint, strongly stabilizing solution of (33) and (34), respectively.

Proof. The proof is a matter of manipulating the formulas, and is a straightforward extension of the proofs of Lemma 9.4.13, Lemma 9.4.14 and Theorem 9.4.15 in [12].

A popular choice in the literature is to use the central controller, which amounts to the choise $L(s) \equiv 0$ in the formula $(54)$, above.

Corollary 4.8 Consider the system $\Sigma\left(A, B, B^{*}, D\right)$ under the assumptions $A 1-A 6$ and let $U$ be finite-dimensional. Let $P, Q, F_{Q}$ and $W$ be as in the previous theorem. The controller

$$
K_{0}(s)=-D-\frac{1}{\sigma^{2}} B^{*} Q\left(s I-A_{0}\right)^{-1} W^{*} P B,
$$

, where $\sigma^{2}=1-\varepsilon^{2}$ and

$$
A_{0}=A+\left(B-\frac{1}{\sigma^{2}} W^{*} P B D\right) F_{Q}-\frac{1}{\sigma^{2}} W^{*} P B B^{*}
$$

stabilizes the system with a robustness margin of $\varepsilon, 0<\varepsilon<\varepsilon_{\max }$.

Finally, we return to our example 2.3.

Example 4.9 We derive a formula for all robustly stabilizing controllers for the flexible beam model of example 2.3. In this example, we had $A^{*}=-A, D=0$, wich makes it easy to see that $P=I, Q=I$. ¿From this, we can see that $\varepsilon_{\max }=\frac{1}{\sqrt{2}}$ and all robustly stabilizing controllers with robustness margin $0<\varepsilon<\varepsilon_{\max }$ are given by

$$
K(s)=\left[\phi_{11}(s) L(s)+\phi_{12}(s)\right]\left[\phi_{21}(s) L(s)+\phi_{22}(s)\right]^{-1},
$$

where

$$
\begin{aligned}
& \left(\begin{array}{ll}
\phi_{11}(s) & \phi_{12}(s) \\
\phi_{21}(s) & \phi_{22}(s)
\end{array}\right)=\left(\begin{array}{cc}
\frac{1}{\varepsilon} I & 0 \\
0 & -\frac{1}{\sigma} I
\end{array}\right) \\
& +\frac{1}{2 \sigma^{2}-1} I\left(\begin{array}{c}
-I \\
I
\end{array}\right) B^{*}\left(s I-A+B B^{*}\right)^{-1} B\left(\begin{array}{ll}
\frac{1}{\varepsilon} I & -\frac{1}{\sigma} I
\end{array}\right) \text {, }
\end{aligned}
$$

$\sigma=\sqrt{1-\varepsilon^{2}}$ and $L \in \mathcal{M} \mathbf{H}_{\infty}$ satisfies $\|L\|_{\infty} \leq 1$.

In particular, the controller $K=-I$, which corresponds with $L(s)=-\frac{\varepsilon}{\sqrt{1-\varepsilon^{2}}} I$, is robustly stabilizing with maximal robustness margin $\frac{1}{\sqrt{2}}$. 


\section{Notation}

Let $Z_{1}, Z_{2}$ be separable Hilbert spaces, $B$ a Banach space and $\Omega \subset \mathbb{C}$.

$\mathcal{L}\left(Z_{1}, Z_{2}\right) \quad$ bounded linear operators from $Z_{1}$ to $Z_{2}$.

$\mathcal{L}\left(Z_{1}\right) \quad \mathcal{L}\left(Z_{1}, Z_{1}\right)$

$\mathbb{C}_{0}^{+} \quad\{s \in \mathbb{C} \mid \operatorname{Re}(s)>0\}$

$\mathbf{L}_{2}(\Omega, B) \quad$ Lebesgue-measurable, square integrable $B$-valued functions on $\Omega$

$\mathbf{H}_{2}(B) \quad$ holomorphic, square integrable, $B$-valued functions on $\mathbb{C}_{0}^{+}$

$\mathbf{L}_{\infty}(B) \quad$ essentially bounded, weakly measurable $B$-valued functions on the imaginary axis

$\mathbf{H}_{\infty}\left[\mathbf{H}_{\infty}\right]^{-1}$ quotient space of $\mathbf{H}_{\infty}(\mathbb{C})$.

$\mathcal{M} \mathbf{H}_{\infty} \quad$ matrices of any size with components in $\mathbf{H}_{\infty}(\mathbb{C})$.

$\mathbf{H}_{\infty}(B) \quad$ bounded, holomorphic $B$-valued functions on $\mathbb{C}_{0}^{+}$.

$r(A) \quad$ spectral radius of $A$

$\mathcal{D}(A) \quad$ domain of $A$

* $\quad A^{*}$, adjoint operator of $A$.

$F^{\dagger}(s):=F(-\bar{s})^{*}$

\section{References}

[1] T. Bailey and J.E. Hubbard jr. Distributed piezoelectric polymer active vibration control of a cantilever beam. AIAA journal on Guidance, Control and Dynamics, pages 605-611, 1985.

[2] A.V. Balakrishnan. Strong stabilizability and the steady state Riccati equation. Applied Mathematics and Optimization, 7:335-345, 1981.

[3] A.V. Balakrishnan. Compensator design for stability enhancement with colocated controllers. IEEE Transactions on Automatic Control, 36:994-1007, 1991.

[4] C.D. Benchimol. A note on weak stabilizability of contraction semigroups. SIAM journal on Control and Optimization, 16:373-379, 1978.

[5] G. Chen, M.C. Delfour, A.M. Krull, and G. Payre. Modelling, stabilization and control of serially connected beams. SIAM journal of Control and Optimization, $25: 526-546,1987$.

[6] R.F. Curtain. Robust stabilizability of normalized coprime factors; the infinitedimensional case. International Journal of Control, 51:1173-1190, 1990.

[7] R.F. Curtain and J.C. Oostveen. The Nehari problem for nonexponentially stable systems. 1997. (submitted). 
[8] R.F. Curtain and J.C. Oostveen. Riccati equations for strongly stabilizable bounded linear systems. 1997. (submitted).

[9] R.F. Curtain and A.J. Pritchard. Robust stabilization of infinite-dimensional systems with respect to coprime-factor perturbations. In D. Elworthy, Everitt, and Lee, editors, Control theory, Dynamical Systems and Geometry of Dynamics, pages 437-456. Marcel Dekker, New York, 1993.

[10] R.F. Curtain and B. van Keulen. Robust control with respect to coprime factors of infinite-dimensional positive real systems. IEEE Transactions on Automatic Control, 37(6):868-871, 1992.

[11] R.F. Curtain, G. Weiss, and M. Weiss. Coprime factorizations for regular linear systems. Automatica, 32:1519-1532, 1996.

[12] R.F. Curtain and H.J. Zwart. An Introduction to Infinite-Dimensional Linear Systems Theory. Springer-Verlag New York, 1995.

[13] R.F. Curtain and H.J. Zwart. Riccati equations and normalized coprime factorizations for strongly stabilizable infinite-dimensional systems. Systems and Control Letters, 28:11-22, 1996.

[14] H. Dym, T.T. Georgiou, and M.C. Smith. Explicit formulas for optimally robust controllers for delay systems. IEEE transactions on automatic control, 40:656-669, 1995.

[15] T.T. Georgiou and M.C. Smith. Optimal robustness in the gap metric. IEEE Transactions on Automatic Control, 35(6):673-685, 1990.

[16] S.M. Joshi. Control of Large Flexible Space Structures, volume 131 of Lecture Notes in Control and Information Sciences. Springer Verlag, Berlin, 1989.

[17] D.C. McFarlane and K. Glover. Robust Controller Design using Normalized Coprime Factor Plant Descriptions, volume 138 of Lecture Notes in Control and Information Sciences. Springer-Verlag, 1989.

[18] A. Pazy. Semigroups of Linear Operators and Applications to Partial Differential Equations. Springer-Verlag, New York, 1983.

[19] S.C. Power. Hankel operators on Hilbert Space. Pitman, 1982.

[20] M. Slemrod. Feedback stabilization of a linear control system in Hilbert space with an a priori bounded control. Mathematics of Control, Signals and Systems, $2: 265-285,1989$.

[21] M.C. Smith. On stabilization and the existence of coprime factorizations. IEEE Transactions on Automatic Control, 34(9):1005-1007, 1989. 